\title{
Understanding the Dipole Moment of Liquid Water from a Self-Attractive Hartree Decomposition
}

Tianyu Zhu, Troy Van Voorhis

Submitted date: 03/12/2020 - Posted date: 03/12/2020

Licence: CC BY-NC-ND 4.0

Citation information: Zhu, Tianyu; Van Voorhis, Troy (2020): Understanding the Dipole Moment of Liquid Water from a Self-Attractive Hartree Decomposition. ChemRxiv. Preprint.

https://doi.org/10.26434/chemrxiv.13184261.v2

The dipole moment of a single water molecule in liquid water has been a critical concept for understanding water's dielectric properties. In this work, we investigate the dipole moment of liquid water through a self-attractive Hartree (SAH) decomposition of total electron density computed by density functional theory, on water clusters sampled from ab initio molecular dynamics simulation of bulk water. By adjusting one parameter that controls the degree of density localization, we reveal two distinct pictures of water dipoles that are consistent with bulk dielectric properties: a localized picture with smaller and less polarizable monomer dipoles, and a delocalized picture with larger and more polarizable monomer dipoles. We further uncover that the collective dipole-dipole correlation is stronger in the localized picture and is key to connecting individual dipoles with bulk dielectric properties. Based on these findings, we suggest considering both individual and collective dipole behaviors when studying the dipole moment of liquid water, and propose new design strategies for developing water models.

File list (2) 


\title{
Understanding the dipole moment of liquid water from a self-attractive Hartree decomposition
}

\author{
Tianyu Zhu, ${ }^{*, \dagger}$ and Troy Van Voorhis*, \\ $\dagger$ Department of Chemistry, Massachusetts Institute of Technology, 77 Massachusetts \\ Avenue, Cambridge, Massachusetts 02139, United States \\ $\ddagger$ Division of Chemistry and Chemical Engineering, California Institute of Technology, \\ Pasadena, California 91125, United States \\ E-mail: tyzhu@caltech.edu; tvan@mit.edu
}




\begin{abstract}
The dipole moment of a single water molecule in liquid water has been a critical concept for understanding water's dielectric properties. In this work, we investigate the dipole moment of liquid water through a self-attractive Hartree (SAH) decomposition of total electron density computed by density functional theory, on water clusters sampled from ab initio molecular dynamics simulation of bulk water. By adjusting one parameter that controls the degree of density localization, we reveal two distinct pictures of water dipoles that are consistent with bulk dielectric properties: a localized picture with smaller and less polarizable monomer dipoles, and a delocalized picture with larger and more polarizable monomer dipoles. We further uncover that the collective dipole-dipole correlation is stronger in the localized picture and is key to connecting individual dipoles with bulk dielectric properties. Based on these findings, we suggest considering both individual and collective dipole behaviors when studying the dipole moment of liquid water, and propose new design strategies for developing water models.
\end{abstract}

\title{
Graphical TOC Entry
}

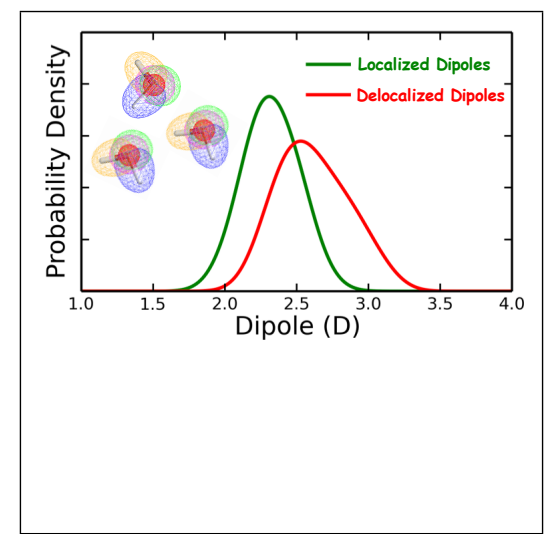


The dipole moment plays a crucial role in determining dielectric properties of liquid water, and is directly related to the intensity of the IR spectrum. For a free water monomer, its dipole moment has been experimentally determined to be 1.855 D. ${ }^{1}$ In water clusters and condensed phases, the dipole moment of individual water molecule is known to be enhanced significantly due to polarization and delocalization effects. ${ }^{2-4}$ Badyal et al. ${ }^{5}$ employed the X-ray diffraction experiment to infer the dipole moment of liquid water to be $2.9 \pm 0.6 \mathrm{D}$ by fitting a charge transfer parameter of $0.5 \pm 0.1 e$ along each $\mathrm{OH}$ bond. However, the monomer dipole in liquid water is an inherently ambiguous concept. In other words, there is no universally true value of the monomer dipole moment in liquid water. Rather, the importance of the monomer dipole moment is that it can quantitatively connect collective fluctuations of a microscopic property (the monomer dipole) to the bulk dielectric properties of the liquid. There are multiple consistent definitions of the monomer dipole and each gives its own form of insight into the bulk properties.

Understanding the connection between individual dipoles and bulk dielectric properties is also essential for developing accurate water models in molecular dynamics simulations, because most force fields are parametrized to reproduce the bulk water properties. Although a wide range of dipole moment values are used in water models ${ }^{6}$ many force fields predict similar bulk dielectric properties. For example, the fixed-charge non-polarizable TIP5P model ${ }^{7}$ and the polarizable iAMOEBA model ${ }^{8}$ both predict the dielectric constant of liquid water to be around 81, despite their distinct parametrizations. Therefore, it is necessary to examine whether there exists specific rules that govern bulk dielectric properties at the microscopic level.

Numerous computational studies have been performed to extract the dipole moment of liquid water. In mixed QM/MM approaches, ${ }^{9-11}$ one water molecule is treated quantum mechanically, which is embedded in water molecules represented by classical point charges and multipole expansions. In this case, the dipole moment of the QM water molecule is straightforwardly taken as the dipole moment of liquid water. On the other hand, full quantum 
chemical calculations of large water clusters and ab initio molecular dynamics simulation of bulk liquid water have been carried out using density functional theory (DFT) or the correlated wavefunction methods. ${ }^{12-19}$ One complexity in these methods is how to recover the concept of a water monomer from the bulk simulations. A common strategy is to transform the wavefunctions of the bulk into the localized molecular orbitals (LMOs), ${ }^{20}$ using methods like Boys localization, ${ }^{21}$ natural bond orbital (NBO) ${ }^{22}$ or maximally localized Wannier functions. ${ }^{23}$ The LMOs are then assigned to nearest water nuclei and each LMO is filled with two electrons. Studies using the LMO scheme predict average monomer dipole moment in liquid water within the range of 2.5-3.1 $\mathrm{D},{ }^{13,14,17,19}$ but no consensus has been reached. Although being computationally efficient, LMOs are normally required to be mutually orthogonal, which may result in unphysical long delocalized tails and overestimated dipole moments.

Unlike wavefunctions, the electron density is a well-defined observable that can be accessed experimentally. Therefore, direct partitioning of the electron density into water monomers is a more natural way to study the dipole moment of liquid water. The commonly used density partitioning method is Bader's quantum theory of atoms in molecules (QTAIM). ${ }^{24}$ Nevertheless, QTAIM divides the electron density into non-overlapping sectors, thus it does not fulfill the fundamental requirement that monomer dipole moments must sum to the overall dipole moment of liquid water. ${ }^{17}$ Alternatively, in this study, we employ a selfattractive Hartree (SAH) decomposition ${ }^{25}$ of the total electron density computed by DFT to extract the monomer dipole moment in liquid water. The SAH decomposition has been demonstrated to be an effective tool to directly unravel chemical bonding from the electron density in molecular complexes. It has also enabled the many-pair expansion ${ }^{26-28}$ calculations in DFT to systematically improve the accuracy of approximate density functionals in molecular systems.

In this work, from a theoretical perspective, we aim to qualitatively understand the monomer dipole moment of liquid water, and reveal how monomer dipoles are correlated to 
form the total dipole moment and produce dielectric properties of the bulk water. In the rest of this paper, we will first describe how SAH decomposition of the electron density allows a clear definition of monomer dipoles inside water clusters with one adjustable parameter that controls the degree of density localization. Then, we will apply the SAH decomposition to analyze realistic liquid water structures to understand how the bulk dielectric properties emerge from the more or less localized monomer dipoles. Finally, we will conclude with suggestions on designing new water models.

The SAH decomposition is devised to partition the electron density into a sum of twoelectron densities (one spin-up and one spin-down) that simultaneously minimize the selfattraction and maintain smooth shapes of fragments. To achieve this goal, one minimizes a regularized Lagrangian

$$
\begin{aligned}
L\left[\left\{\phi_{i}\right\}, \gamma(\mathbf{r}),\left\{\epsilon_{i}\right\}\right]=-\alpha \cdot \frac{1}{2} \sum_{i}^{N} \iint \frac{\rho_{i}(\mathbf{r}) \rho_{i}\left(\mathbf{r}^{\prime}\right)}{\left|\mathbf{r}-\mathbf{r}^{\prime}\right|} d \mathbf{r} d \mathbf{r}^{\prime}+\sum_{i}^{N} \int\left|\nabla \phi_{i}(\mathbf{r})\right|^{2} d \mathbf{r} \\
\quad+\int \gamma(\mathbf{r})\left(\sum_{i}^{N} \rho_{i}(\mathbf{r})-\rho_{T}(\mathbf{r})\right) d \mathbf{r}-\sum_{i}^{N} \epsilon_{i}\left(\int \rho_{i}(\mathbf{r}) d \mathbf{r}-2\right),
\end{aligned}
$$

with $\rho_{i}(\mathbf{r})=2\left|\phi_{i}(\mathbf{r})\right|^{2}$ being the two-electron densities, $\phi_{i}(\mathbf{r})$ as auxiliary orbitals, $\rho_{T}$ as the input total density, and $\gamma(\mathbf{r})$ and $\left\{\epsilon_{i}\right\}$ as Lagrangian multipliers. From now on, we will refer $\left\{\rho_{i}(\mathbf{r})\right\}$ as pair densities. The first term of Eq. 1 is the sum of self-attraction energies, and the second term is the sum of kinetic energies of localized pair densities. Because simultaneous minimization of the self-attraction and kinetic energy are conflicting requirements, minimization of their sum would give fragment densities that are localized but still smooth. This method can also be understood as a regularized Edmiston-Ruedenberg localization. ${ }^{29}$ The parameter $\alpha$ in Eq. 1 is introduced to control the extent of localization of the resulting pair densities (i.e., the larger $\alpha$ produces more localized pair densities). This is a particularly attractive feature in this study, as it allows us to investigate how delocalization effects contribute to the magnitude of partitioned water dipoles by adjusting $\alpha$. The third and fourth terms in Eq. 1 refer to two constraints: (1) the pair densities sum to the total 
density; and (2) each pair density integrates to 2. These two constraints clearly indicates that the SAH decomposition is a density partition scheme. Because the SAH pair densities are constrained to sum to the input total density, all multipoles of the full system are exactly sums of the multipoles of the pair densities.

Minimizing this Lagrangian $\left(\frac{\delta L}{\delta \phi_{i}}=0\right)$ results in the following set of equations:

$$
\left[-\frac{1}{2} \nabla^{2}-2 \alpha \int \frac{\phi_{i}\left(\mathbf{r}^{\prime}\right)^{2}}{\left|\mathbf{r}-\mathbf{r}^{\prime}\right|} d \mathbf{r}^{\prime}+\gamma(\mathbf{r})\right] \phi_{i}(\mathbf{r})=\epsilon_{i} \phi_{i}(\mathbf{r})
$$

which we solve using a constrained self-consistent field (SCF) algorithm. We refer the readers to Ref. ${ }^{25}$ for the detailed SAH algorithm. From Eq. 2, one important feature of SAH is that the resulting pair densities are ground-state densities of some potential and therefore nodeless.

To illustrate how we define monomer dipoles in water clusters, we first applied the SAH decomposition to a water tetramer cluster. The geometry of the water tetramer was optimized using the second-order perturbation theory (MP2) ${ }^{30}$ in the aug-cc-pVTZ basis. ${ }^{31,32}$ The total electron density of the water tetramer was then computed using DFT with the B3LYP functional, ${ }^{33}$ in a series of uncontracted aug-cc-pVXZ $(\mathrm{X}=\mathrm{D}, \mathrm{T}, \mathrm{Q})$ basis. The basis functions with highest angular momentum $(g)$ were removed from the aug-cc-pVQZ basis for the sake of computational efficiency. All calculations were carried out in QChem 4.2 software package. ${ }^{34}$ The B3LYP total density was partitioned into pair densities using the SAH decomposition, with a series of $\alpha$ values $(\alpha=0.2,0.5,1,1.5,2,3)$. The resulting pair densities $\left\{\rho_{i}(\mathbf{r})\right\}$ were assigned to their nearest water nuclei, with 5 pair densities constituting one water monomer. The dipole moment of monomer $M$ was then calculated as

$$
\vec{\mu}_{M}=-2 \sum_{i \in M}\left\langle\phi_{i}|\mathbf{r}| \phi_{i}\right\rangle+\sum_{A \in M} Z_{A} \mathbf{R}_{A}
$$

where we used the relation $\rho_{i}(\mathbf{r})=2\left|\phi_{i}(\mathbf{r})\right|^{2}$ and $\phi_{i}(\mathbf{r})$ is the auxiliary orbital in the SAH decomposition. $Z_{A}$ and $\mathbf{R}_{A}$ are the charge and position of nucleus $A(=\mathrm{O}, \mathrm{H})$ in monomer $M$. 
Since the SAH density of each monomer has exactly 10 electrons, the SAH-decomposed water molecule is neutral and its dipole moment is gauge-invariant and well-defined. There exist multiple views of water monomers in liquid water, one of which allows intermolecular charge transfer and non-neutral water monomers. ${ }^{35-37}$ However, this view would make it impossible to unambiguously define the quantity of dipole moment, thus we stick to assigning exactly 10 electrons to each water molecule.
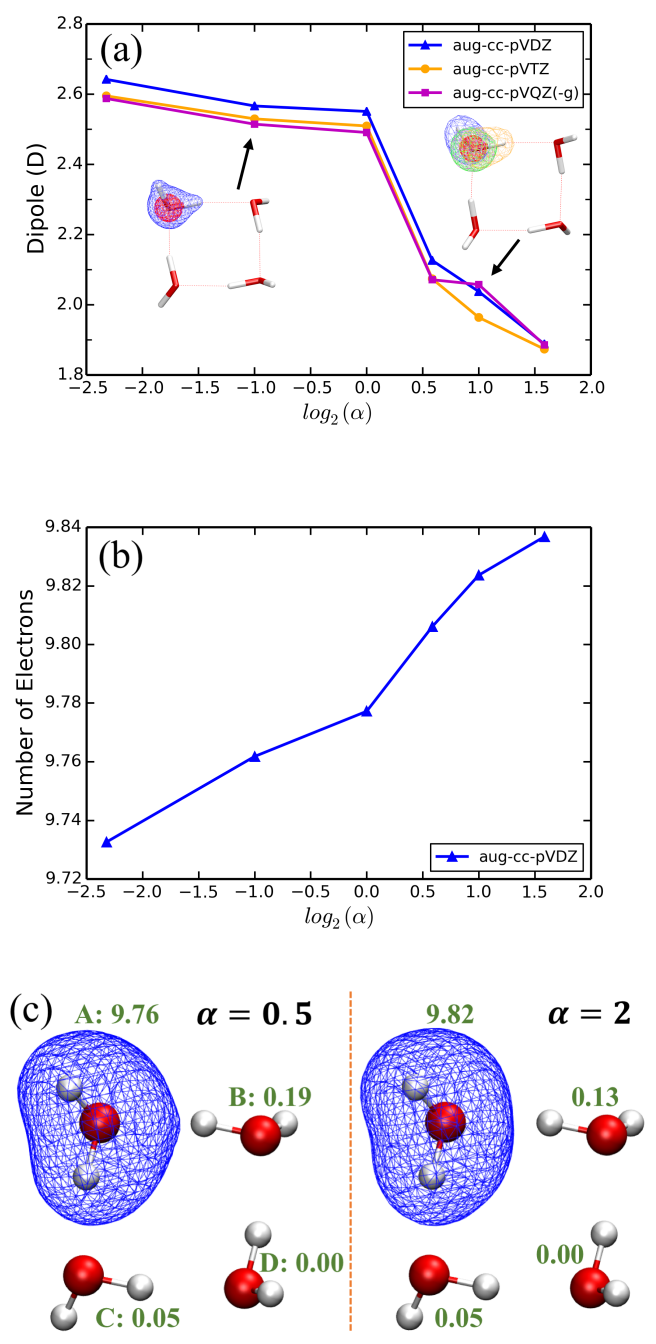

Figure 1: (a) Dipole moment of a water monomer in the water tetramer from the SAH decomposition, as a function of localization parameter $\alpha$. (b) Hirshfeld population analysis of a water monomer's electron density restricted to its own atomic basis functions. (c) Hirshfeld population of a water monomer's electron density on atomic basis functions of all water molecules. 
The SAH dipole moment results are shown in Fig. 1a. When using a small localization parameter $\alpha(\alpha=0.2)$, the molecular dipole moment in the water tetramer is computed to be around $2.6 \mathrm{D}$, substantially larger than the dipole moment of a free water monomer $(1.855$ D). As the density partitioning becomes more localized (as $\alpha$ increases), the molecular dipole moment in the water tetramer decreases, and is only around $1.9 \mathrm{D}$ at $\alpha=3$. We also observe an abrupt drop of the dipole moment value in the range of $\alpha=1.0 \sim 1.5$. To understand these unexpected behaviors, we plot the decomposed pair densities in Fig. 1a. Two distinct density partitioning patterns are found for $\alpha \leq 1.0$ and $\alpha \geq 1.5$. For $\alpha \leq 1.0$, although the pair densities are localized in the sense that they belong to only one water monomer, they are delocalized over that one water molecule. As shown in Fig. 1a, for each water monomer at $\alpha=0.5$, there are one O-1s core-like pair density (red) and four pair densities (blue) resembling the total density of a water molecule. On the other hand, for each water monomer at $\alpha \geq 1.5$, the pair densities are localized enough to unravel the chemical bonding within each water molecule. In addition to the O-1s core-like pair density, two O-H bonding pair densities and two oxygen lone pair densities are also revealed, which is more consistent with the valence bond theory. This change in density partitioning patterns causes the abrupt reduction of the dipole moment in Fig. 1a. We note that since the SAH decomposition is a density partitioning scheme, it does not suffer from numerical instability due to diffuse basis functions like localized molecular orbital methods (see Fig. S4 for a comparison between cc-pVDZ and aug-cc-pVDZ basis sets).

To further study how delocalization effect influences the dipole moment enhancement in the water tetramer, we performed the Hirshfeld population analysis ${ }^{38}$ of a water monomer's electron density and counted the number of electrons residing on its own atomic basis functions using the Multiwfn package. ${ }^{39}$ By definition, each SAH-decomposed water monomer has exactly 10 electrons. However, as we used the atomic basis of the whole water tetramer to expand the partitioned fragment densities, it is expected that each water monomer's density would integrate to less than 10 electrons on its own atom-centered basis. As demonstrated in 
Fig. 1b, more localized density partitioning leads to more electrons (closer to $10 \mathrm{e}$ ) residing on each water molecule's own basis. This result suggests charge delocalization (or charge transfer) between water molecules indeed accounts for great amount of dipole moment enhancement. Comparing Fig. 1a and Fig. 1b, a small amount of $0.1 e$ charge delocalization difference can lead to $0.7 \mathrm{D}$ dipole moment difference in the water tetramer. In Ref., ${ }^{17} \mathrm{a}$ similar effect of delocalization tails on the monomer dipole moments in water clusters has been studied using the truncated Magnasco-Perico method.

In Fig. 1c, we further discuss how the 10 electrons of water monomer $\mathrm{A}$ are distributed over the atomic basis functions of all water molecules. At $\alpha=0.5,9.76 e$ of electrons reside on monomer A's own basis functions, while $0.19 e$ and $0.05 e$ of electrons reside on basis functions of monomer $\mathrm{B}$ and monomer $\mathrm{C}$. When $\alpha$ is increased to 2 , there is a 0.06 $e$ increase of electrons localizing on monomer A $(9.82 e)$ and a $0.06 e$ decrease of electrons locating on monomer B $(0.13 \mathrm{e})$. This analysis indicates that the reduction of dipole moment in the water tetramer as $\alpha$ increases is mainly due to the more localized electron density distribution along the proton-accepting hydrogen bond.

We then investigated the dipole moment of liquid water using the SAH decomposition. We started by performing ab initio molecular dynamics (AIMD) simulation of a cubic cell with 64 water molecules in the NVT ensemble $\left(298 \mathrm{~K}, 1.0 \mathrm{~g} / \mathrm{cm}^{-3}\right)$ using the CP2K software package. ${ }^{40}$ The revPBE-D3 density functional ${ }^{41,42}$ was employed with the GTH-MOLOPTDZVP-SR basis ${ }^{43}$ and GTH-PBE pseudopotential. ${ }^{44,45}$ After an equilibration of 10 ps (with a time step of $0.5 \mathrm{fs}$ ), we carried out a production run of $20 \mathrm{ps}$. The oxygen-oxygen radial distribution function $g_{\mathrm{OO}}(r)$ was computed and shown to agree well with the experimental results $^{46,47}$ in Fig. S1, confirming the accuracy of our AIMD simulation.

Using liquid water structures sampled from the AIMD trajectory, we first studied how monomer dipole moment is enhanced as the number of surrounding water molecules increases. We randomly picked one water molecule in one AIMD snapshot as the "center" molecule, and successively added its surrounding water molecules according to the O-O distances. The 

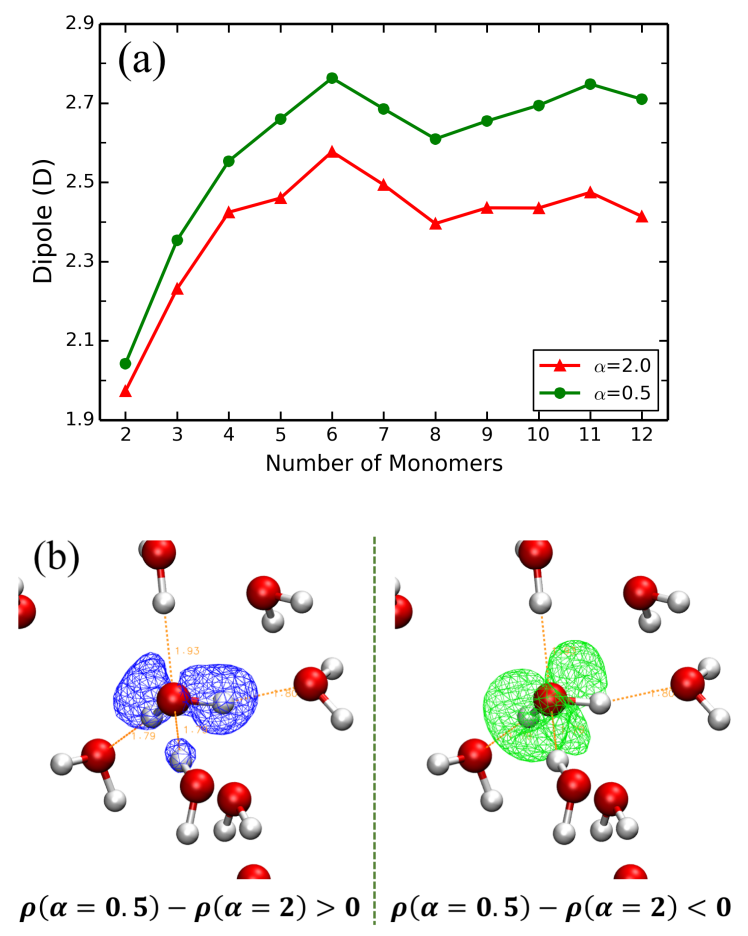

Figure 2: (a) The dipole moment of the central water molecule with increasing number of total water molecules, calculated using two localization parameters $(\alpha=0.5$ and 2$)$ in the SAH decomposition. The water geometries were sampled from one liquid water AIMD snapshot. (b) Electron density difference $(\rho(\alpha=0.5)-\rho(\alpha=2))$ on the central water molecule between two localization schemes. An isosurface value of 0.004 a.u. is used.

electron densities of this series of water clusters were calculated using DFT with the B3LYP functional and uncontracted aug-cc-pVDZ basis. After applying the SAH decomposition ( $\alpha=0.5$ and 2 ) to total electron densities of these water clusters, the dipole moment of central water molecule was computed and presented in Fig. 2a. Already in the water dimer, there is a difference of $0.1 \mathrm{D}$ in the dipole moment of central water monomer between the $\alpha=0.5$ and $\alpha=2 \mathrm{SAH}$ results. As the number of surrounding water molecules increases, two trends are revealed. First, the monomer dipole moment rapidly increases to $2.8 \mathrm{D}$ $(\alpha=0.5)$ or $2.6 \mathrm{D}(\alpha=2)$, and starts to plateau after the total number of monomers reaches 6 . Second, the less localized $\alpha=0.5$ density partitioning always gives greater dipole moment, and the difference between the two $(\alpha=0.5$ and $\alpha=2)$ becomes more prominent in larger water clusters. We also notice that in Fig. $2 \mathrm{a}$, the $\alpha=2 \mathrm{SAH}$ dipole moment is only 
$0.2 \sim 0.3 \mathrm{D}$ less than the $\alpha=0.5$ result in the bulk water structures, significantly smaller than the $\sim 0.6 \mathrm{D}$ reduction in the water tetramer case (Fig. 1a). This finding indicates that the polarization effect accounts for a larger fraction of dipole moment enhancement in the bulk water than in the quasi-two-dimensional water tetramer structure.

To understand how electron density distributes differently at $\alpha=0.5$ and $\alpha=2$, we further plot the electron density difference $(\rho(\alpha=0.5)-\rho(\alpha=2))$ of the central water molecule in Fig. $2 \mathrm{~b}$ using the water decamer geometry. We find that at $\alpha=0.5$, the electron density is more delocalized along both proton-accepting and proton-donating hydrogen bonds. On the other hand, at $\alpha=2$, the electron density is more localized around the water molecule and less delocalized along the directions of hydrogen bonds.

To obtain a meaningful distribution of molecular dipole moment in liquid water, we performed the same analysis on 5 central water molecules in 20 snapshots sampled from the final 20 ps AIMD trajectory (i.e., 100 water cluster geometries in total). As the central molecular dipole moment quickly plateaus with respect to the cluster size (Fig. 2a), we fixed the water cluster size to 10 water molecules. Gaussian-smoothed distributions of the dipole moment of central water molecule are presented in Fig. 3a, where each data point is replaced by a Gaussian function with a width of $0.12 \mathrm{D}$. As can be seen, the distribution of central molecular dipole moment is very broad, ranging from $2.0 \mathrm{D}$ to $3.3 \mathrm{D}$ in the $\alpha=0.5 \mathrm{SAH}$ decomposition, with a mean water dipole moment of $2.63 \mathrm{D}$.

Consistent with the analysis in Fig. 2a, we find that the mean dipole moment of the central water molecule is $0.28 \mathrm{D}$ larger in the $\alpha=0.5$ density partitioning than the more localized $\alpha=2 \mathrm{SAH}$ result. This difference partly quantifies the contribution of charge delocalization to the enhancement of dipole moment in liquid water. We also observe a broader dipole distribution using $\alpha=0.5$, with a standard deviation of $0.21 \mathrm{D}$, compared to the standard deviation of $0.15 \mathrm{D}$ using $\alpha=2$. As the broadness of the dipole distribution reflects the tendency of charge polarization, we infer from the standard deviation that the less localized density partitioning also predicts larger polarizability in water molecules, in 

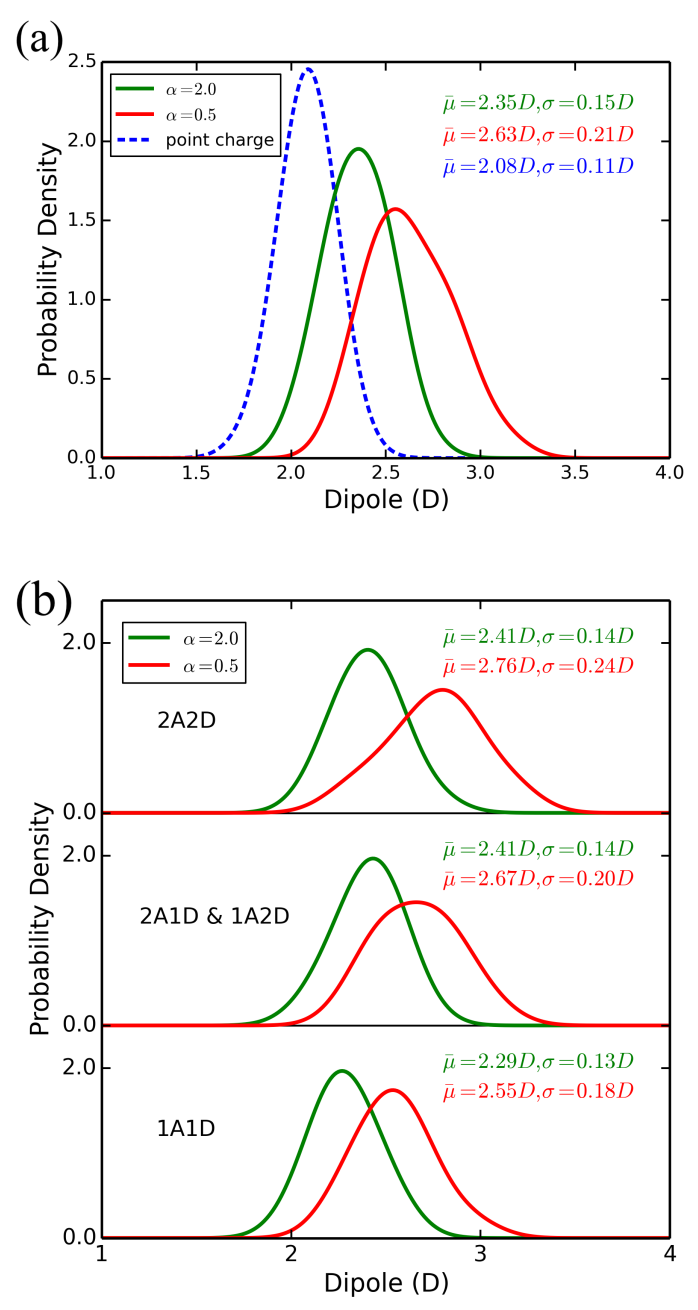

Figure 3: (a) Distributions of the dipole moment of central water molecule in 100 water decamer geometries sampled from the AIMD trajectory, calculated using two localization parameters $(\alpha=0.5$ and 2). (b) Distributions of the dipole moment of central water molecule with different hydrogen bond networks. "A" and "D" refer to accepting and donating protons in hydrogen bonds.

addition to greater magnitude of dipoles.

In Fig. 3b, we further classify the dipole moment distributions according to different hydrogen bond networks around the central water molecule. Following Ref., ${ }^{48}$ two water molecules are considered to form a hydrogen bond if their O-O distance is less than $3.5 \AA$, and the $\mathrm{O}-\mathrm{H} \cdots \mathrm{O}$ angle is less than $30^{\circ}$. Depending on whether the central water molecule accepts (A) or donates (D) a proton in a hydrogen bond (HB), we collect statistics for "2A2D" (4 HBs), "2A1D \& 1A2D" (3 HBs), and "1A1D" (2 HBs). We find that at $\alpha=0.5$, 
the mean dipole moment and standard deviation of the delocalized water dipoles increase from 2.55/0.18 D in "1A1D" to 2.76/0.24 D in "2A2D" as the number of HB increases, in agreement with previous computational studies. ${ }^{49}$ However, at $\alpha=2$, the localized water dipole distribution shows a weaker dependence on the number of HB. As a result, the dipole moment and polarizability differences between two water dipole pictures $(\alpha=0.5$ and $\alpha=2)$ become more prominent as the number of HB grows. Due to the limited sample size of our calculation, we do not conduct the comparison between "2A1D" and "1A2D" HB networks and will investigate this issue in the future work.

The finding in Fig. 3 is surprising, because it suggests that two distinct water models, one with larger dipole and polarizability and the other with smaller dipole and polarizability, can be derived from exactly the same charge distribution, and yield the same bulk dielectric properties. To understand this rather counterintuitive result, we further computed the radial dipole-dipole spatial correlation function ${ }^{50}$ between SAH water dipoles inside all 100 sampled water decamers:

$$
F(r)=\frac{\sum_{i j} P_{i j}(r)\left[\hat{\mu}_{i} \cdot \hat{\mu}_{j}\right]}{\sum_{i j} P_{i j}(r)},
$$

where $\hat{\mu}_{i}$ and $\hat{\mu}_{j}$ are the normalized dipole moment of monomer $i$ and monomer $j$ within the same water decamer. $P_{i j}(r)$ is a Gaussian probability distribution with a width of 0.3 $\AA$ centered on the O-O distance $r_{i j}$ between monomer $i$ and monomer $j$. The computed radial dipolar correlation functions for SAH water dipoles are presented in Fig. 4.

The dipole-dipole correlation functions of $\alpha=0.5$ and $\alpha=2$ SAH dipoles show similar trends, where the correlation function $F(r)$ reaches the minimum as the O-O distance increases to $4.0 \sim 4.5 \AA$ and has a second peak between $5.5 \sim 6.0 \AA$. This trend is in qualitative agreement with previous dipolar correlation studies of liquid water, ${ }^{50}$ but is not quantitative because we only considered dipoles inside water decamers. Comparing the $\alpha=0.5$ and $\alpha=2 \mathrm{SAH}$ results, one sees that the more localized $\alpha=2$ density partitioning yields stronger dipole-dipole correlation function over all distances shown in Fig. 4. To the best of our knowledge, this enhanced dipole-dipole correlation has not been observed before and is 


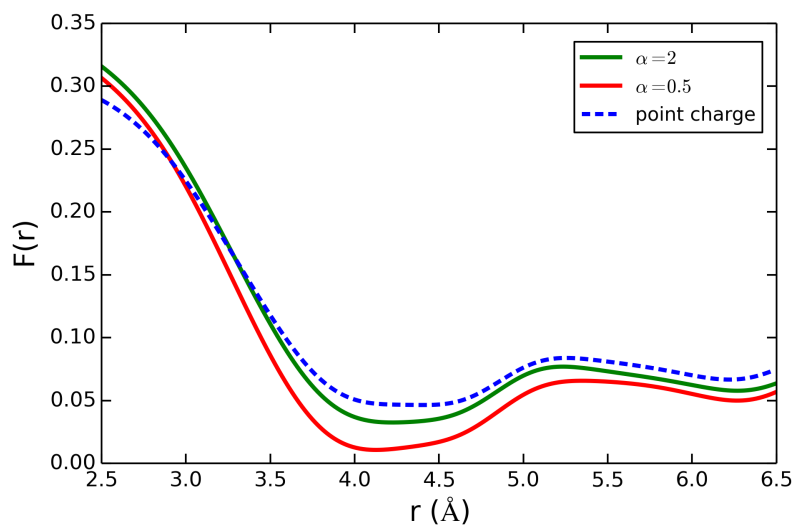

Figure 4: Radial dipole-dipole spatial correlation functions for SAH water dipoles in 100 sampled water decamers, with localization parameters $\alpha=0.5$ and $\alpha=2$.

not captured by any known fixed-charge or polarizable water force field. This result also explains the counterintuitive finding that two distinct SAH water models predict the same bulk dielectric properties. Although the dipole moment and polarizability of a water monomer is smaller in the more localized $(\alpha=2)$ density partitioning, the intermolecular dipole-dipole correlation is stronger. This enhanced collective effect between water dipoles compensates for the smaller individual magnitudes, giving rise to the same bulk property as in the less localized $(\alpha=0.5)$ SAH partitioning.

To build a preliminary quantitative relation between the SAH decomposition and classical atomic-site water models, we further investigate how a 3-site fixed-charge water model can produce the same bulk dielectric properties as the SAH-decomposed pair densities. Here, we assume a 3 -site water model with $q_{\mathrm{O}}=-2 q_{\mathrm{H}}\left(\right.$ similar to TIP3P ${ }^{51}$ ) and compute the monomer dipoles as $\vec{\mu}_{M}=\sum_{A \in M} q_{A} \mathbf{R}_{A}$. To be consistent with the SAH decomposition results, the point-charge water dipoles are computed on the 100 water decamer structures sampled from the AIMD trajectory. The point charge parameter $q_{\mathrm{O}}$ is fitted to be $-0.723 e$ so that the mean error of the total dipole moment (compared to $\mathrm{SAH} \alpha=2$ and $\alpha=0.5$ ) of 100 water decamers is zero. As seen in Fig. 3a, in order to reproduce the same bulk property (total dipole moment), the mean value $(2.08 \mathrm{D})$ and standard deviation $(0.11 \mathrm{D})$ of the dipole moment distribution predicted by the point-charge model are both smaller than 
the SAH results. This smaller individual dipole moment and polarizability are compensated by the stronger collective dipole-dipole correlation function in the point-charge model at all O-O distances greater than $3.3 \AA$, as shown in Fig. 4. This observation suggests that fixedcharge models with correlated dipole orientations may be a promising route for developing new water force fields. Meanwhile, we point out that the strong dipole-dipole correlation for the point-charge model in this study is provided by the revPBE-D3 AIMD water structures. It would be interesting to explore strategies to design such dipole correlations directly using the force field in the future.

In this work, we investigated the monomer dipole moment of liquid water through a selfattractive Hartree decomposition. Two distinct pictures of water dipoles have been uncovered by tuning the degree of density localization in the SAH decomposition: a localized picture where the monomer dipole moment and polarizability is smaller, and a delocalized picture with larger monomer dipole moment and polarizability. We have further revealed that the strength of collective dipole-dipole correlation varies in these two pictures, which leads to the same bulk dielectric properties despite different individual dipole behaviors. Thus, instead of a strict definition, we suggest a more comprehensive way to understand the dipole moment in liquid water. In addition to the magnitude and distribution of individual dipole moments, one should also consider the collective behavior between water dipoles, which may be quantified by the dipole-dipole correlation function used above.

This work also suggests new design strategies for water models in molecular dynamics simulations. Based on our findings, two natural routes for designing water force fields that reproduce bulk dielectric properties have emerged: fixed-charge models where the dipole orientations are correlated, and polarizable models where the dipole orientations are largely independent. While the latter strategy has been actively pursued and resulted in promising polarizable water models, ${ }^{8,52-55}$ the former strategy has not been attempted yet. In the future, it would be attractive to derive a water model with fixed dipoles that still captures the enhanced angular correlations of the dipoles from the localized SAH-decomposed densities. 


\section{Acknowledgement}

This work was funded by a grant from the NSF CHE-1900358. We thank Piotr de Silva for inspiring discussions.

\section{Supporting Information Available}

Oxygen-oxygen radial distribution function $g_{O O}(r)$ from the AIMD simulation, SAH density decomposition error analysis for Fig. 2, distributions of the dipole moments of all water molecules in 100 sampled water decamers, and numerical test of dependence on basis sets.

\section{References}

(1) Clough, S. A.; Beers, Y.; Klein, G. P.; Rothman, L. S. Dipole moment of water from Stark measurements of H2O, HDO, and D2O. J. Chem. Phys. 1973, 59, 2254-2259.

(2) Gregory, J. K.; Clary, D. C.; Liu, K.; Brown, M. G.; Saykally, R. J. The water dipole moment in water clusters. Science 1997, 275, 814-817.

(3) Coulson, C. A.; Eisenbergf, D. Interactions of H2O molecules in ice I. The dipole moment of an H2O molecule in ice. Proc. R. Soc. London. Ser. A. 1966, 291, 445-453.

(4) Batista, E. R.; Xantheas, S. S.; Jónsson, H. Molecular multipole moments of water molecules in ice Ih. J. Chem. Phys. 1998, 109, 4546-4551.

(5) Badyal, Y. S.; Saboungi, M. L.; Price, D. L.; Shastri, S. D.; Haeffner, D. R.; Soper, A. K. Electron distribution in water. J. Chem. Phys. 2000, 112, 9206-9208.

(6) Chaplin, M. http://www.Isbu.ac.uk/water/water_models.html. 
(7) Mahoney, M. W.; Jorgensen, W. L. A five-site model for liquid water and the reproduction of the density anomaly by rigid, nonpolarizable potential functions. J. Chem. Phys. 2000, 112, 8910-8922.

(8) Wang, L. P.; Head-Gordon, T.; Ponder, J. W.; Ren, P.; Chodera, J. D.; Eastman, P. K.; Martinez, T. J.; Pande, V. S. Systematic improvement of a classical molecular model of water. J. Phys. Chem. B 2013, 117, 9956-9972.

(9) Kongsted, J.; Osted, A.; Mikkelsen, K. V.; Christiansen, O. Dipole and quadrupole moments of liquid water calculated within the coupled cluster/molecular mechanics method. Chem. Phys. Lett. 2002, 364, 379-386.

(10) Georg, H. C.; Canuto, S. Electronic properties of water in liquid environment. A sequential QM/MM study using the free energy gradient method. J. Phys. Chem. B 2012, $116,11247-11254$.

(11) Bakó, I.; Mayer, I. Hierarchy of the collective effects in water clusters. J. Phys. Chem. A 2016, 120, 631-638.

(12) Laasonen, K.; Sprik, M.; Parrinello, M.; Car, R. "Ab initio" liquid water. J. Chem. Phys. 1993, 99, 9080-9089.

(13) Silvestrelli, P. L.; Parrinello, M. Water molecule dipole in the gas and in the liquid phase. Phys. Rev. Lett. 1999, 82, 3308-3311.

(14) Kemp, D. D.; Gordon, M. S. An interpretation of the enhancement of the water dipole moment due to the presence of other water molecules. J. Phys. Chem. A 2008, 112, $4885-4894$.

(15) Wang, Y.; Huang, X.; Shepler, B. C.; Braams, B. J.; Bowman, J. M. Flexible, ab initio potential, and dipole moment surfaces for water. I. Tests and applications for clusters up to the 22-mer. J. Chem. Phys. 2011, 134, 094509. 
(16) Willow, S. Y.; Salim, M. A.; Kim, K. S.; Hirata, S. Ab initio molecular dynamics of liquid water using embedded-fragment second-order many-body perturbation theory towards its accurate property prediction. Sci. Rep. 2015, 5, 14358.

(17) Bakó, I.; Mayer, I. On dipole moments and hydrogen bond identification in water clusters. J. Phys. Chem. A 2016, 120, 4408-4417.

(18) Liu, J.; He, X.; Zhang, J. Z. Structure of liquid water-a dynamical mixture of tetrahedral and 'ring-and-chain' like structures. Phys. Chem. Chem. Phys. 2017, 19, 11931-11936.

(19) Ruiz Pestana, L.; Mardirossian, N.; Head-Gordon, M.; Head-Gordon, T. Ab initio molecular dynamics simulations of liquid water using high quality meta-GGA functionals. Chem. Sci. 2017, 8, 3554-3565.

(20) Jensen, F. Introduction to computational chemistry; John Wiley \& Sons, 2017.

(21) Foster, J.; Boys, S. Canonical configurational interaction procedure. Rev. Mod. Phys. 1960, 32, 300 .

(22) Reed, A. E.; Curtiss, L. A.; Weinhold, F. Intermolecular interactions from a natural bond orbital, donor-acceptor viewpoint. Chem. Rev. 1988, 88, 899-926.

(23) Marzari, N.; Mostofi, A. A.; Yates, J. R.; Souza, I.; Vanderbilt, D. Maximally localized Wannier functions: Theory and applications. Rev. Mod. Phys. 2012, 84, 1419-1475.

(24) Bader, R. F. A quantum theory of molecular structure and its applications. Chem. Rev. 1991, 91, 893-928.

(25) Zhu, T.; de Silva, P.; Van Voorhis, T. Self-attractive Hartree decomposition: Partitioning electron density into smooth localized fragments. J. Chem. Theory Comput. 2018, $14,92-103$. 
(26) Zhu, T.; de Silva, P.; van Aggelen, H.; Van Voorhis, T. Many-electron expansion: A density functional hierarchy for strongly correlated systems. Phys. Rev. B 2016, 93, 201108.

(27) de Silva, P.; Zhu, T.; Van Voorhis, T. Long-range interactions from the many-pair expansion: A different avenue to dispersion in DFT. J. Chem. Phys. 2017, 146, 024111.

(28) Zhu, T.; de Silva, P.; Van Voorhis, T. Implementation of the many-pair expansion for systematically improving density functional calculations of molecules. J. Chem. Theory Comput. 2019, 15, 1089-1101.

(29) Edmiston, C.; Ruedenberg, K. Localized atomic and molecular orbitals. Rev. Mod. Phys. 1963, 35, 457.

(30) Møller, C.; Plesset, M. S. Note on an approximation treatment for many-electron systems. Phys. Rev. 1934, 46, 618.

(31) Dunning Jr, T. H. Gaussian basis sets for use in correlated molecular calculations. I. The atoms boron through neon and hydrogen. J. Chem. Phys. 1989, 90, 1007-1023.

(32) Kendall, R. A.; Dunning Jr, T. H.; Harrison, R. J. Electron affinities of the first-row atoms revisited. Systematic basis sets and wave functions. J. Chem. Phys. 1992, 96, 6796-6806.

(33) Becke, A. D. Density-functional thermochemistry. III. The role of exact exchange. $J$. Chem. Phys. 1993, 98, 5648-5652.

(34) Shao, Y.; Gan, Z.; Epifanovsky, E.; Gilbert, A. T. B.; Wormit, M.; Kussmann, J.; Lange, A. W.; Behn, A.; Deng, J.; Feng, X. et al. Advances in molecular quantum chemistry contained in the Q-Chem 4 program package. Mol. Phys. 2015, 113, 184215. 
(35) Ben-Amotz, D. Unveiling electron promiscuity. J. Phys. Chem. Lett. 2011, 2, 12161222.

(36) Vácha, R.; Marsalek, O.; Willard, A. P.; Bonthuis, D. J.; Netz, R. R.; Jungwirth, P. Charge transfer between water molecules as the possible origin of the observed charging at the surface of pure water. J. Phys. Chem. Lett. 2012, 3, 107-111.

(37) Torii, H. Extended nature of the molecular dipole of hydrogen-bonded water. J. Phys. Chem. A 2013, 117, 2044-2051.

(38) Hirshfeld, F. L. Bonded-atom fragments for describing molecular charge densities. Theor. Chem. Acc. 1977, 44, 129-138.

(39) Lu, T.; Chen, F. Multiwfn: A multifunctional wavefunction analyzer. J. Comput. Chem. 2012, 33, 580-592.

(40) Kühne, T. D.; Iannuzzi, M.; Del Ben, M.; Rybkin, V. V.; Seewald, P.; Stein, F.; Laino, T.; Khaliullin, R. Z.; Schütt, O.; Schiffmann, F. et al. CP2K: An electronic structure and molecular dynamics software package - Quickstep: Efficient and accurate electronic structure calculations. J. Chem. Phys. 2020, 152, 194103.

(41) Zhang, Y.; Yang, W. Comment on "generalized gradient approximation made simple". Phys. Rev. Lett. 1998, 80, 890.

(42) Grimme, S.; Antony, J.; Ehrlich, S.; Krieg, H. A consistent and accurate ab initio parametrization of density functional dispersion correction (DFT-D) for the 94 elements H-Pu. J. Chem. Phys. 2010, 132, 154104.

(43) VandeVondele, J.; Hutter, J. Gaussian basis sets for accurate calculations on molecular systems in gas and condensed phases. J. Chem. Phys. 2007, 127, 114105.

(44) Goedecker, S.; Teter, M.; Hutter, J. Separable dual-space Gaussian pseudopotentials. Phys. Rev. B 1996, 54, 1703. 
(45) Hartwigsen, C.; Goedecker, S.; Hutter, J. Relativistic separable dual-space Gaussian pseudopotentials from H to Rn. Phys. Rev. B 1998, 58, 3641.

(46) Soper, A. K. The radial distribution functions of water and ice from 220 to $673 \mathrm{~K}$ and at pressures up to $400 \mathrm{MPa}$. Chem. Phys. 2000, 258, 121-137.

(47) Skinner, L. B.; Huang, C.; Schlesinger, D.; Pettersson, L. G.; Nilsson, A.; Benmore, C. J. Benchmark oxygen-oxygen pair-distribution function of ambient water from x-ray diffraction measurements with a wide Q-range. J. Chem. Phys. 2013, 138, 074506.

(48) Luzar, A.; Chandler, D. Effect of environment on hydrogen bond dynamics in liquid water. Phys. Rev. Lett. 1996, 76, 928-931.

(49) Bakó, I.; Daru, J.; Pothoczki, S.; Pusztai, L.; Hermansson, K. Effects of H-bond asymmetry on the electronic properties of liquid water - An AIMD analysis. J. Mol. Liq. 2019, 293, 111579.

(50) Zhang, C.; Galli, G. Dipolar correlations in liquid water. J. Chem. Phys. 2014, 141, 084504 .

(51) Jorgensen, W. L.; Chandrasekhar, J.; Madura, J. D.; Impey, R. W.; Klein, M. L. Comparison of simple potential functions for simulating liquid water. J. Chem. Phys. 1983, 79, 926-935.

(52) Ren, P.; Ponder, J. W. Polarizable atomic multipole water model for molecular mechanics simulation. J. Phys. Chem. B 2003, 10\%, 5933-5947.

(53) Lamoureux, G.; MacKerell, A. D.; Roux, B. A simple polarizable model of water based on classical drude oscillators. J. Chem. Phys. 2003, 119, 5185-5197.

(54) Fanourgakis, G. S.; Xantheas, S. S. Development of transferable interaction potentials for water. V. Extension of the flexible, polarizable, Thole-type model potential (TTM3- 
F, v. 3.0) to describe the vibrational spectra of water clusters and liquid water. J. Chem. Phys. 2008, 128, 074506.

(55) Babin, V.; Leforestier, C.; Paesani, F. Development of a "first principles" water potential with flexible monomers: Dimer potential energy surface, VRT spectrum, and second virial coefficient. J. Chem. Theory Comput. 2013, 9, 5395-5403. 


\title{
Supporting information for:
}

\section{Understanding the dipole moment of liquid} water from a self-attractive Hartree decomposition

\author{
Tianyu Zhu, ${ }^{*, \dagger}$ and Troy Van Voorhis ${ }^{*, \dagger}$ \\ $\dagger$ Department of Chemistry, Massachusetts Institute of Technology, 77 Massachusetts \\ Avenue, Cambridge, Massachusetts 02139, United States \\ $\ddagger$ Division of Chemistry and Chemical Engineering, California Institute of Technology, \\ Pasadena, California 91125, United States \\ E-mail: tyzhu@caltech.edu; tvan@mit.edu
}




\section{AIMD oxygen-oxygen radial distribution function}

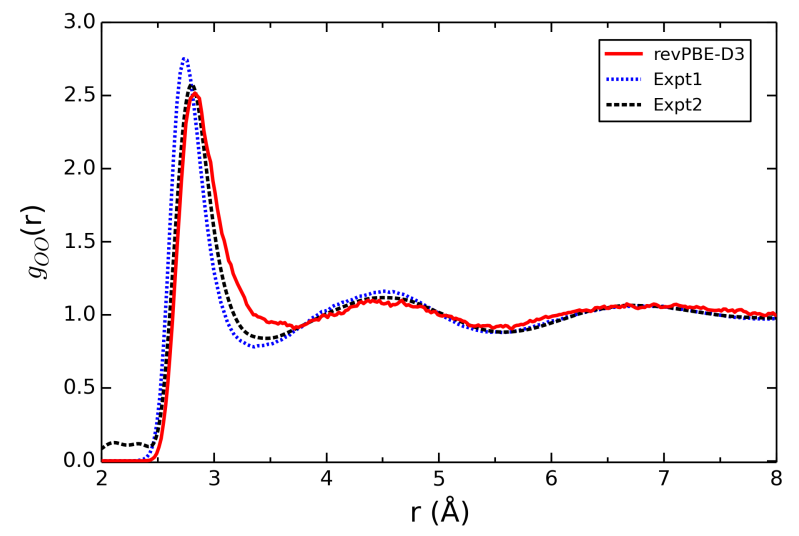

Figure S1: Oxygen-oxygen radial distribution function $g_{\mathrm{OO}}(r)$ of liquid water computed from the AIMD simulation using the revPBE-D3 functional, compared to experimental results. "Expt1" is taken from Ref., ${ }^{1}$ and "Expt2" is taken from Ref. ${ }^{2}$

\section{SAH density decomposition error analysis}

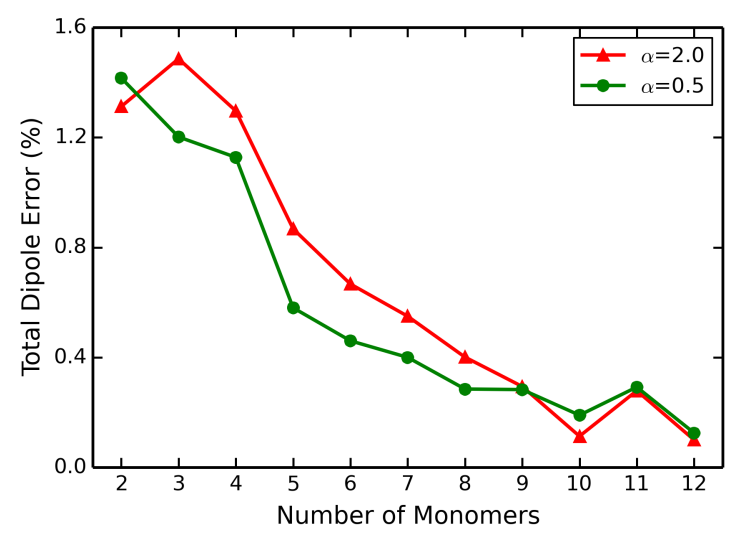

Figure S2: Numerical (percentage) errors of the total dipole moment of water clusters from the SAH decomposition with respect to the input density, using two localization parameters ( $\alpha=0.5$ and $\alpha=2$ ), with increasing number of total water molecules, The water cluster geometries are the same as in Fig. 2 of the main text.

The SAH decomposition is a density partition scheme, where the SAH pair densities are constrained to sum to the input total density. Thus, in theory, the dipole of the full system 
is exactly sums of the dipoles of the pair densities. In practice, because we implemented the SAH decomposition in finite Gaussian basis, the density constraints are satisfied subject to numerical errors. In Fig. S2, we demonstrate the numerical errors of the total dipole moment of water clusters computed from the SAH decomposition, with increasing number of total water molecules. One can see that even the largest percentage errors are within 1.6\%. The errors decrease with increasing number of total water molecules, possibly due to more auxiliary Gaussian basis functions that better constrain the density decomposition. As our main conclusions are drawn from water decamer structures, the expected numerical errors on total dipole moments are below $0.4 \%$.

\section{Dipole moment distributions of all water molecules in sam-}

\section{pled water decamers}

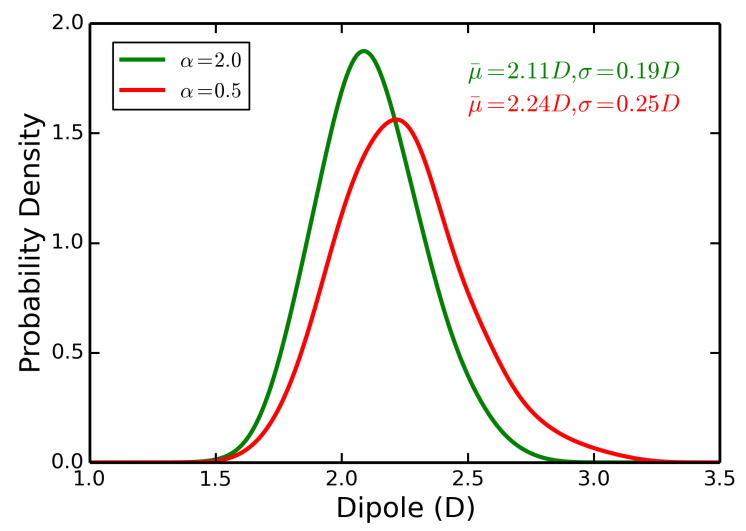

Figure S3: Distributions of the dipole moment of all water molecules in 100 water decamer geometries sampled from the AIMD trajectory, calculated using two localization parameters $(\alpha=0.5$ and 2$)$ in the SAH decomposition.

In Fig. S3, we show the distributions of the dipole moment of all water molecules in 100 water decamers sampled from the AIMD trajectory, which are the same structures used in Fig. 3 of the main text. We find the same trend as in Fig. 3, i.e., the more localized decomposition yields smaller average dipole moment and polarizability. However, the differ- 
ence between the two distributions are not as obvious as in Fig. 3, where only the central water molecule is considered. This is because the "edge" water molecules are not fully surrounded by other water molecules, and are less polarized and have smaller degree of charge delocalization.

\section{Numerical test of dependence on basis sets}

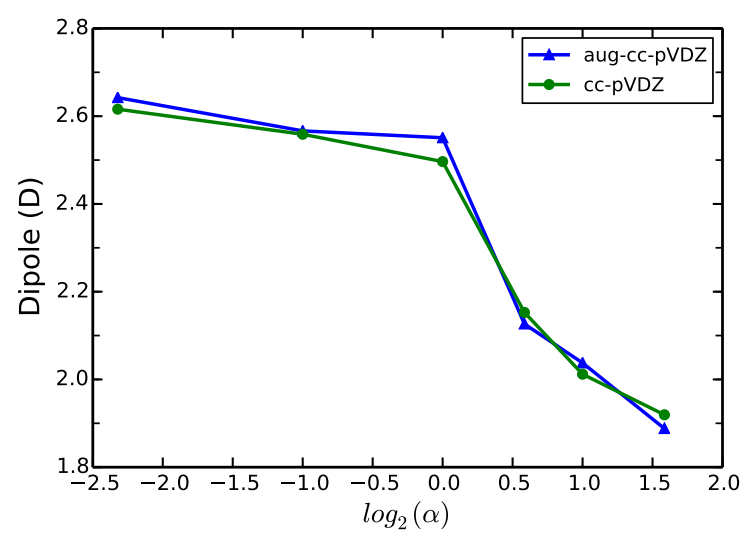

Figure S4: Dipole moment of a water monomer in the water tetramer from the SAH decomposition, using the uncontracted aug-cc-pVDZ and cc-pVDZ basis sets.

Since the SAH decomposition is a density partitioning scheme, it does not suffer from numerical instability due to diffuse basis functions like localized molecular orbital methods. To confirm this point, we perform SAH decomposition for the water tetramer in the uncontracted cc-pVDZ basis, and compare the dipole moment results to the uncontracted aug-cc-pVDZ basis, as shown in Fig. S4. The cc-pVDZ results follow the same trend as the aug-cc-pVDZ results, and the dipole moment difference between the two basis sets is within $0.05 \mathrm{D}$. Therefore, the results in this paper do not depend on the augmentation of basis function. 


\section{References}

(1) Soper, A. K. The radial distribution functions of water and ice from 220 to $673 \mathrm{~K}$ and at pressures up to $400 \mathrm{MPa}$. Chem. Phys. 2000, 258, 121-137.

(2) Skinner, L. B.; Huang, C.; Schlesinger, D.; Pettersson, L. G.; Nilsson, A.; Benmore, C. J. Benchmark oxygen-oxygen pair-distribution function of ambient water from x-ray diffraction measurements with a wide Q-range. J. Chem. Phys. 2013, 138, 074506. 
